\title{
Interval Valued D-Implications
}

R.H.S. REISER ${ }^{1}$, Programa de Pós-Graduação em Informática, UCPel, 96010-000 Pelotas, RS, Brasil.

B.C. BEDREGAL 2 , R.H.N. SANTIAGO 3 , Depto de Informática e Matemática Aplicada, UFRN, 59072-970 Natal, RN, Brasil.

G.P. DIMURO 4 , Programa de Pós-Graduação em Modelagem Computacional, Campus Carreiros, 96201-090 Rio Grande, RS, Brasil.

\begin{abstract}
The aim of this work is to introduce the concepts of interval Dimplications and automorphisms, analyzing their main properties and establishing the relation between them. Also, interval D-implications are related with punctual D-implications and automorphisms.
\end{abstract}

Keywords. Interval Fuzzy Logics, Interval Fuzzy Implications, Interval D-implications.

\section{Introduction}

Fuzzy set theory [37] is the oldest and most widely reported component of presentday soft computing, which deals with the design of flexible information processing systems [25], with applications in control systems [14], decision making [13], expert systems [35], pattern recognition [25], etc. On the other hand, Interval Mathematics [26] is a mathematical theory that aims at the representation of uncertain input data and parameters, and the automatic and rigorous control of the errors which arise in numerical computations.

The interval-valued fuzzy set theory, which aims at the integration of Fuzzy Theory and Interval Mathematics, has been studied from different viewpoints (see, e.g., $[15,17,18,27,29,38])$. One of these approaches uses membership functions with interval values, in order to model the uncertainty in the process of determining exact membership grades with the usual fuzzy membership functions. Then, to each element of the universe a closed subinterval of the unit interval is assigned, approximating the membership degree.

This work adopts the approach introduced in $[8,9]$, where interval extensions of fuzzy connectives are constructed as their interval representations [34], considering both correctness (accuracy) and optimality aspects as required in [20]. This

\footnotetext{
${ }^{1}$ reiser@ucpel.tche.br

2bedregal@dimap.ufrn.br

${ }^{3}$ regivan@dimap.ufrn.br

4 gracaliz@gmail.com
} 
approach was also considered in our previous works $[5,6,7,31,32]$. In fuzzy set theory, implication functions are usually derived from t-norms and t-conorms in several ways, e.g, S-implications, R-implications, QL-implications and D-implications. ${ }^{5}$ The importance of implications is not only because they are used in representing "If ... then" rules in fuzzy systems, but also because they are use in performing inferences in approximate reasoning and fuzzy control. This is the main reason for searching many different models to perform this kind of fuzzy connectives. In particular, D-implications were studied only recently (see, e.g., [23, 24]).

The aim of this work is to introduce interval D-implications and to study their relationship with interval automorphisms, and also with punctual D-implications and automorphisms. This paper is organized as follows. In Sect. 2., we review the main concepts related to interval representations. Interval fuzzy t-conorms ( $\mathrm{t}$ norms) and negations are presented in sections 3. and 4., respectively. Interval fuzzy implications and D-implications are discussed in Sect. 5.. Interval automorphisms are presented in Sect. 6., and their relationship with interval D-implications in Sect. 7.. Section 8. is the Conclusion.

\section{Interval Representations}

Let $\mathbb{U}=\{[a, b] \mid 0 \leq a \leq b \leq 1\}$ be the set of subintervals of $U=[0,1] \subseteq \mathbb{R}$. For $X=[\underline{X}, \bar{X}] \in \mathbb{U}$, the projections $l, r: \mathbb{U} \rightarrow U$ are defined by

$$
l(X)=\underline{X} \text { and } r(X)=\bar{X} .
$$

Among the partial orders that may be defined on $\mathbb{U}[12]$, in this work we consider: - Product order: $X \leq Y \Leftrightarrow \underline{X} \leq \underline{Y}$ and $\bar{X} \leq \bar{Y}$.

- Inclusion order: $X \subseteq Y \Leftrightarrow \underline{X} \geq \underline{Y}$ and $\bar{X} \leq \bar{Y}$.

Definition 2.1. $F: \mathbb{U}^{n} \longrightarrow \mathbb{U}$ is an interval representation of a function $f$ : $U^{n} \longrightarrow U$ if, for each $\vec{X} \in \mathbb{U}^{n}$ and $\vec{x} \in \vec{X}, f(\vec{x}) \in F(\vec{X})$. [34]

$F: \mathbb{U}^{n} \longrightarrow \mathbb{U}$ is a better interval representation of $f: U^{n} \longrightarrow U$ than $G:$ $\mathbb{U}^{n} \longrightarrow \mathbb{U}$, denoted by $G \sqsubseteq F$, if, for each $\vec{X} \in \mathbb{U}^{n}, F(\vec{X}) \subseteq G(\vec{X})$.

Definition 2.2. [34] The best interval representation of a real function $f, f: U^{n} \longrightarrow$ $U$, is the interval function $\widehat{f}: \mathbb{U}^{n} \longrightarrow \mathbb{U}$, defined by

$$
\widehat{f}(\vec{X})=[\inf \{f(\vec{x}) \mid \vec{x} \in \vec{X}\}, \sup \{f(\vec{x}) \mid \vec{x} \in \vec{X}\}]
$$

The interval function $\widehat{f}$ is well defined and for any other interval representation $F$ of $f$, it holds that $F \sqsubseteq \widehat{f}$, that is, $\widehat{f}$ returns a narrower interval than any other interval representation of $f$. Thus, $\hat{f}$ has the optimality property of interval algorithms [20], when it is seen as an algorithm to compute a real function $f$.

\footnotetext{
${ }^{5}$ There are also some other methods of generating fuzzy implications (see, e.g, [36]).
} 


\section{Interval t-norms and t-conorms}

A $t$-conorm $(t$-norm $)$ is a function $S(T): U^{2} \rightarrow U$ that is commutative, associative, monotonic and has 0 (1) as neutral element.

Example 1. Typical examples of t-norms and t-conorms, respectively, are the following:

1. $T_{M}(x, y)=\min \{x, y\}$ and $S_{M}(x, y)=\max \{x, y\}$

2. $T_{P}(x, y)=x y$ and $S_{P}(x, y)=x+y-x y$;

3. $T_{L}(x, x)=\max (x+y-1,0)$ and $S_{L}(x, y)=\min (x+y, 1)$;

In the following, we present the interval generalizations of t-conorms (t-norms), and also two results provided in [8, 10].

Definition 3.1. $\mathbb{S}: \mathbb{U}^{2} \rightarrow \mathbb{U}$ is an interval t-conorm (t-norm) if it is commutative, associative, monotonic w.r.t. the product and inclusion order and $[0,0]([1,1])$ is the neutral element.

Proposition 3.1. If $S$ (T) is a t-conorm (t-norm) then $\widehat{S}: \mathbb{U}^{2} \rightarrow \mathbb{U}\left(\widehat{T}: \mathbb{U}^{2} \rightarrow \mathbb{U}\right)$ is an interval t-conorm (t-norm). Characterizations of $\widehat{S}$ and $\widehat{T}$ are given, respectively, by

$$
\begin{aligned}
\widehat{S}(X, Y) & =[S(\underline{X}, \underline{Y}), S(\bar{X}, \bar{Y})], \\
\widehat{T}(X, Y) & =[T(\underline{X}, \underline{Y}), T(\bar{X}, \bar{Y})] .
\end{aligned}
$$
by

Considering $F: \mathbb{U}^{n} \rightarrow \mathbb{U}$, we define the functions $\underline{F}, \bar{F}: U^{n} \rightarrow U$, respectively

$$
\begin{aligned}
\underline{F}\left(x_{1}, \ldots, x_{n}\right) & =l\left(F\left(\left[x_{1}, x_{1}\right], \ldots,\left[x_{n}, x_{n}\right]\right)\right) \\
\bar{F}\left(x_{1}, \ldots, x_{n}\right) & =r\left(F\left(\left[x_{1}, x_{1}\right], \ldots,\left[x_{n}, x_{n}\right]\right)\right) .
\end{aligned}
$$

Proposition 3.2. Let $\mathbb{S}$ be an interval t-conorm, $\mathbb{T}$ an interval $t$-norm and the functions $\underline{\mathbb{S}}, \overline{\mathbb{S}}$ and $\mathbb{T}, \overline{\mathbb{T}}$ t-conorms and $t$-norms, respectively. Then, for all $X, Y \in \mathbb{U}$, it holds that:

$$
\begin{aligned}
& \mathbb{S}(X, Y)=[\underline{\mathbb{S}}(\underline{X}, \underline{Y}), \overline{\mathbb{S}}(\bar{X}, \bar{Y})], \\
& \mathbb{T}(X, Y)=[\underline{\mathbb{T}}(\underline{X}, \underline{Y}) \overline{\mathbb{T}}(\bar{X}, \bar{Y})],
\end{aligned}
$$

Example 2. Based on Propositons 3.1 and 3.2, some interval t-norms and interval t-conorms are now considered:

1. $\widehat{T_{M}}(X, Y)=[\min (\underline{X}, \underline{Y}), \min (\bar{X}, \bar{Y})]=\inf \{X, Y\}=\mathbb{T}_{M}(X, Y) ;$ and $\widehat{S_{M}}(X, Y)=[\max (\underline{X}, \underline{Y}), \max (\bar{X}, \bar{Y})]=\sup \{X, Y\}=\mathbb{S}_{M}(X, Y)$.

2. $\widehat{T_{P}}(X, Y)=[\underline{X Y}, \widehat{X Y}]=\mathbb{T}_{P}(X, Y)$, and $\widehat{S_{P}}(X, Y)=[\underline{X}+\underline{Y}-\underline{X Y}, \bar{X}+\bar{Y}-\overline{X Y}]=\mathbb{S}_{P}(X, Y) ;$

3. $\widehat{T_{L}}(X, Y)=[\max (\underline{X}+\underline{Y}-1,0), \max (\bar{X}+\bar{Y}-1,0)]=\sup (X+Y-[1 ; 1],[0 ; 0])=\mathbb{T}_{L}(X, Y)$, $\widehat{S_{L}}(X, Y)=[\min (\underline{X}+\underline{Y}, 1), \min (\bar{X}+\bar{Y}, 1)]=\sup (X+Y,[1 ; 1])=\mathbb{S}_{L}(X, Y)$. Thus,

$$
\widehat{T_{L}}(X, Y)= \begin{cases}{[\underline{X}+\underline{Y}-1, \bar{X}+\bar{Y}-1],} & \text { if } \underline{X}+\underline{Y} \geq 1, \\ {[0,0],} & \text { if } \overline{\bar{X}} \leq 1, \\ {[0, \bar{X}+\bar{Y}-1],} & \text { otherwise; }\end{cases}
$$




$$
\widehat{S_{L}}(X, Y)= \begin{cases}{[\underline{X}+\underline{Y}, \bar{X}+\bar{Y}],} & \text { if } \bar{X}+\bar{Y} \leq 1, \\ {[1,1]} & \text { if }, \underline{X}+\underline{Y} \geq 1, \\ \mathbb{T}_{L}(X, Y)=[\underline{X}+\underline{Y}, 1], & \text { otherwise. }\end{cases}
$$

\section{Interval Fuzzy Negation}

A function $N: U \rightarrow U$ is a fuzzy negation if

N1 : $N(0)=1$ and $N(1)=0$;

N2 : If $x \geq y$ then $N(x) \leq N(y), \forall x, y \in I$.

Fuzzy negations satisfying the involutive property are called strong fuzzy negations [21]:

N3 : $N(N(x))=x, \forall x \in U$.

Definition 4.1. An interval function $\mathbb{N}: \mathbb{U} \longrightarrow \mathbb{U}$ is an interval fuzzy negation if, for any $X, Y$ in $\mathbb{U}$, the following properties hold:

$\mathbb{N} 1: \mathbb{N}([0,0])=[1,1]$ and $\mathbb{N}([1,1])=[0,0]$;

$\mathbb{N} 2$ : If $X \geq Y$ then $\mathbb{N}(X) \leq \mathbb{N}(Y)$;

$\mathbb{N} 3$ : If $X \subseteq Y$ then $\mathbb{N}(X) \subseteq \mathbb{N}(Y)$.

If $\mathbb{N}$ also meets the involutive property, it is said to be a strong interval fuzzy negation:

$\mathbb{N} 4: \mathbb{N}(\mathbb{N}(X))=X, \forall X \in \mathbb{U}$.

Let $N: U \longrightarrow U$ be a fuzzy negation. A characterization of $\widehat{N}$ is given by:

$$
\widehat{N}(X)=[N(\bar{X}), N(\underline{X})] .
$$

Proposition 4.1. $\mathbb{N}: \mathbb{U} \longrightarrow \mathbb{U}$ is an interval strong fuzzy negation if and only if there exists a strong fuzzy negation $N$ such that $\mathbb{N}=N$ [2].

Clearly, in this case, it holds that $N=\underline{\mathbb{N}}=\overline{\mathbb{N}}$.

Example 3. The interval extension of the standard fuzzy negation $N_{C}(x)=1-x$ is characterized by the expression $\mathbb{N}(X)=[1,1]-X=[1-\bar{X}, 1-\underline{X}]=\widehat{N_{C}}(X)$.

\section{Interval D-Implications}

Several definitions for fuzzy implications together with related properties have been given (see, e.g., $[1,11,16,33,36]$ ), where a binary function $I: U^{2} \longrightarrow U$ is a fuzzy implication if, at least, $I$ satisfies the minimal boundary conditions: $I(1,1)=$ $I(0,1)=I(0,0)=1$ and $I(1,0)=0$. However, other different properties may be required. This paper also considers the following three properties of fuzzy implications: 
I1a : If $x \leq z$ then $I(x, y) \geq I(z, y)$;

I1b : If $y \leq z$ then $I(x, y) \leq I(x, z)$;

I2 : $I(1, x)=x$.

Let $S$ be a t-conorm, $N$ be a strong fuzzy negation and $T$ be a t-norm. A Dishkant Implication (D-implication, for short) is a fuzzy implication defined, for all $x, y \in[0,1]$, by $[23,24]$ :

$$
I_{S, T, N}(x, y)=S(T(N(x), N(y)), y) .
$$

Example 4. In the following, basic fuzzy D-implications are considered:

1. $I_{S_{L}, T_{M}, N_{C}}(x, y)=\min (1-x+y, 1)$ (called Lukaziewski implication).

2. $I_{T_{P}, S_{P}, N_{C}}(x, y)=y+(1-x)(1-y)^{2}$.

3. $I_{S_{L}, T_{L}, N_{C}}(x, y)=\max (1-x, y)$ (called Kleene-Dienes implication).

Definition 5.1. $\mathbb{I}: \mathbb{U}^{2} \longrightarrow \mathbb{U}$ is an interval fuzzy implication if the next conditions hold:

$\mathbb{I}([1,1],[1,1])=\mathbb{I}([0,0],[0,0])=\mathbb{I}([0,0],[1,1])=[1,1] \quad$ and $\quad \mathbb{I}([1,1],[0,0])=[0,0]$.

The properties I1a, I1b and I2 of fuzzy implications can be naturally extended:

I1 a : If $X \leq Z$ then $\mathbb{I}(X, Y) \geq \mathbb{I}(Z, Y)$;

$\mathbb{I} 1 \mathbf{b}:$ If $Y \leq Z$ then $\mathbb{I}(X, Y) \leq \mathbb{I}(X, Z)$;

$\mathbb{I} 2: \mathbb{I}([1,1], X)=X$.

The proofs of the three following propositions follow directly from the definition of $\widehat{I}$. From a fuzzy implication, it is possible to obtain an interval fuzzy implication canonically:

Proposition 5.1. If I is a fuzzy implication then $\widehat{I}$ is an interval fuzzy implication. tion:

We can recover the original fuzzy implication from its best interval representa-

Proposition 5.2. If I is a fuzzy implication then, for each $x, y \in U$, it holds that $I(x, y)=l(\widehat{I}([x, x],[y, y]))=r(\widehat{I}([x, x],[y, y]))$.

In the following proposition, the best interval representation of a fuzzy implication is shown to be an inclusion-monotonic function.

Proposition 5.3. Let $I$ be a fuzzy implication. For each $X_{1}, X_{2}, Y_{1}, Y_{2} \in \mathbb{U}$, if $X_{1} \subseteq X_{2}$ and $Y_{1} \subseteq Y_{2}$ then it holds that $\widehat{I}\left(X_{1}, Y_{1}\right) \subseteq \widehat{I}\left(X_{2}, Y_{2}\right)$.

Theorem 5.1. If $\mathbb{I}: \mathbb{U}^{2} \rightarrow \mathbb{U}$ is an inclusion monotonic interval fuzzy implication satisfying $\mathbb{I} 1 a$ and $\mathbb{I} 1 b$, then $\mathbb{I}$ and $\overline{\mathbb{I}}$ are fuzzy implications, which satisfy the properties $\mathbf{I 1} \mathbf{1}, \mathbf{I 1} \mathbf{b}$ and such that $\mathbb{I}(X, Y)=[\underline{\mathbb{I}}(\bar{X}, \underline{Y}), \overline{\mathbb{I}}(\underline{X}, \bar{Y})]$. 
Proof. See [4].

Given an interval t-conorm $\mathbb{S}$, an interval t-norm $\mathbb{T}$ and a strong interval negation $\mathbb{N}$ the function

$$
\mathbb{I}_{\mathbb{S}, \mathbb{T}, \mathbb{N}}(X, Y)=\mathbb{S}(\mathbb{T}(\mathbb{N}(X), \mathbb{N}(Y)), Y) .
$$

is said an interval D-implication.

Theorem 5.2. Let $S$ be a t-conorm, $T$ be a t-norm and $N$ be a fuzzy negation. If $S, T$ and $N$ are continuous then

$$
\mathbb{I}_{\widehat{S}, \widehat{T}, \widehat{N}}=\widehat{I_{S, T, N}} \text {. }
$$

Proof. Considering $X, Y \in \mathbb{U}$, one has that

$$
\begin{aligned}
\mathbb{I}_{\widehat{S}, \widehat{T}, \widehat{N}}(X, Y) & =\widehat{S}(\widehat{T}((\widehat{N}(X), \widehat{N}(Y)), Y)) \text { by Eq. }(5.2) \\
& =\widehat{S}(\widehat{T}([N(\bar{X}), N(\underline{X})],[N(\bar{Y}), N(\underline{Y})]), Y) \text { by Eq. }(4.1) \\
& =\widehat{S}([T(N(\bar{X}), N(\bar{Y})), T(N(\underline{X}), N(\underline{Y}))], Y) \text { by Eq. }(3.2) \\
& =[S(T(N(\bar{X}), N(\bar{Y})), \underline{Y}), S(T(N(\underline{X}), N(\underline{Y})), \bar{Y})] \text { by Eq. }(3.1)
\end{aligned}
$$

Since, for each $x \in X$ and $y \in Y, S(T(N(\bar{X}), N(\bar{Y})), \underline{Y}) \leq S(T(N(x, y)), y) \leq$ $S(T(N(\underline{X}), N(\underline{Y})), \bar{Y})$, then it holds that $\widehat{I_{S, T, N}}(X, Y) \subseteq \mathbb{I}_{\widehat{S}, \widehat{T}, \widehat{N}}(X, Y)$. On the other hand, if $z \in \widehat{S}(\widehat{T}(N(X), N(Y)), Y)$, then, by the continuity of $S$, there exist $z_{1} \in \widehat{T}(N(X), N(Y))$ and $z_{2} \in Y$ such that $S\left(z_{1}, z_{2}\right)=z$. Thus, by the continuity of $N$ and $T$, there exist $z_{1 a} \in X, z_{1 b} \in Y$ such that $T\left(N\left(z_{1 a}\right), N\left(z_{1 b}\right)\right)=z_{1}$ and, thus, it holds that $S\left(T\left(N\left(z_{1 a}\right), N\left(z_{1 b}\right)\right), z_{2}\right)=z$. If $z_{2} \leq z_{1 a}$, then $\left.S\left(T\left(N\left(z_{2}\right), N\left(z_{1 b}\right)\right), z_{2}\right),\right)$ $\geq z$, and by the commutativity of $T$, it holds that $\left.S\left(T\left(N\left(z_{1 b}\right), N\left(z_{2}\right)\right), z_{2}\right),\right) \geq z$. In addition, if $z_{1 b} \leq z_{2}$, then it is valid that $\left.S\left(T\left(N\left(z_{1 a}\right), N\left(z_{1 b}\right)\right), z_{1 b}\right),\right) \leq z$. So, one has that $z \in\left[I_{S, T, N}\left(z_{1 a}, z_{1 b}\right), I_{S, T, N}\left(z_{1 b}, z_{2}\right)\right] \subseteq\left\{I_{S, N, T}(x, y) \mid x \in X, y \in Y\right\}$, and $\left\{I_{S, T, N}(x, y) \mid x \in X, y \in Y\right\} \subseteq \widehat{I_{S, T, N}}(X, Y)$. Therefore, it follows that $\mathbb{I}_{\widehat{S}, \widehat{T}, \widehat{N}}(X, Y)=\widehat{S}(\widehat{T}(\widehat{N}(X), \widehat{N}(Y)), Y) \subseteq \widehat{I_{S, T, N}}(X, Y)$.

Corollary 5.1. If I is a continuous D-Implication then $\widehat{I}$ is an interval D-implication.

Proof. It is straightforward, following from Theorem 5.2.

Example 5. Based on Theorem 5.2, interval extensions of implications in Example 4 can be given by the following expressions:

$1 . \mathbb{I}_{\widehat{S_{L}}, \widehat{T_{M}}, \widehat{N_{C}}}(X, Y)=[\min (\min (1-\bar{X}, 1-\bar{Y})+\underline{Y}, 1), \min (\min (1-\underline{X}, 1-\underline{Y})+\bar{Y}, 1)]$, that means

$$
\begin{aligned}
& I_{S_{L}, T_{M}, N_{C}}(X, Y)=\mathbb{I}_{\widehat{S_{L}}, \widehat{T_{M}}, \widehat{N_{C}}}(X, Y)= \begin{cases}{[1-\bar{Y}+\underline{Y}, 1],} & \text { if } \bar{X} \leq \bar{Y} ; \\
{[1-\bar{X}+\underline{Y}, 1],} & \text { if } \underline{X} \leq \bar{Y} \leq \bar{X} ; \\
{[1-\bar{X}+\underline{Y}, 1-\underline{X}+\bar{Y}],} & \text { otherwise. }\end{cases} \\
& 2 . \mathbb{I}_{\widehat{S_{P}}, \widehat{T_{P}}, \widehat{N_{C}}}(X, Y)=[(1-\bar{X})(1-\bar{Y})(1-\underline{Y})+\underline{Y},(1-\underline{X})(1-\bar{Y})(1-\underline{Y})+\bar{Y}]=I_{S_{P}, T_{P}, N_{C}}(X, Y) . \\
& 3 . \mathbb{I}_{\widehat{S_{L}}, \widehat{T_{L}}, \widehat{N_{C}}}(X, Y)=[\min (\max (1-\bar{X}-\bar{Y}, 0)+\underline{Y}, 1), \min (\max (1-\underline{X}-\underline{Y})+\bar{Y}, 1)] .
\end{aligned}
$$


Thus, $\mathbb{I}_{\widehat{S_{L}}, \widehat{T_{L}}, \widehat{N_{C}}}(X, Y)=\widehat{I_{S_{L}, T_{L}, N_{C}}}(X, Y)$, and

$\mathbb{I}_{\widehat{S_{L}}, \widehat{T_{L}}, \widehat{N_{C}}}(X, Y)= \begin{cases}{[1-\bar{X}-\bar{Y}+\underline{Y}, 1-\underline{X}-\underline{Y}+\bar{Y}],} & \text { if } \bar{Y} \leq \underline{X}+\underline{Y} \leq \bar{X}+\bar{Y} \leq 1 ; \\ {[1-\bar{X}-\bar{Y}+\underline{Y}, 1],} & \text { if } \underline{X}+\underline{Y} \leq \bar{Y} \leq \bar{X}+\bar{Y} \leq 1 ; \\ {[\underline{Y}, 1-\underline{X}-\underline{Y}+\bar{Y}],} & \text { if } \underline{X}+\underline{Y} \leq \bar{Y} \leq 1 \leq \bar{X}+\bar{Y} ; \\ {[\underline{Y}, \bar{Y}],} & \text { otherwise. }\end{cases}$

Proposition 5.4. If $\mathbb{I}$ is an interval D-implication then the properties $\mathbb{I} 1 a$ and $\mathbb{I} 2$ hold.

Proof. Consider $\mathbb{I}$ as an interval D-implication and $X, Y, Z \in \mathbb{U}$.

I1 1 : Based on the monotonicity of the interval t-norm $\mathbb{T}$, the interval t-conorm $\mathbb{S}$ and interval negation $\mathbb{N}$, if $X \leq Z$ then $\mathbb{S}(\mathbb{T}(\mathbb{N}(X), \mathbb{N}(Y)), Y) \geq \mathbb{S}(\mathbb{T}(\mathbb{N}(Z), \mathbb{N}(Y)), Y)$. Therefore, it holds that $\mathbb{I}_{\mathbb{S}, \mathbb{T}, \mathbb{N}}(X, Y) \geq \mathbb{I}_{\mathbb{S}, \mathbb{T}, \mathbb{N}}(Z, Y)$.

$\mathbb{I} 2$ : One has that $\mathbb{I}_{\mathbb{S}, \mathbb{T}, \mathbb{N}}([1,1], Y)=\mathbb{S}(\mathbb{T}(\mathbb{N}([1,1]), \mathbb{N}(Y)), Y)=\mathbb{S}(\mathbb{T}([0,0], \mathbb{N}(Y)), Y)$. By the monotonicity of $\mathbb{T}$, it holds that $\mathbb{S}(\mathbb{T}([0,0], \mathbb{N}(Y)), Y)=\mathbb{S}([0,0], Y)=Y$. Then, it follows that $\mathbb{I}_{\mathbb{S}, \mathbb{T}, \mathbb{N}}([1,1], Y)=Y$.

Denote by $\mathcal{C}(S), \mathcal{C}(T), \mathcal{C}(I)$ and $\mathcal{C}(N)$ the classes of continuous t-conorms, tnorms and D-implications, and strong fuzzy negations, respectively. The related interval extensions are indicated by $\mathcal{C}(\mathbb{S}), \mathcal{C}(\mathbb{T}), \mathcal{C}(\mathbb{I})$ and $\mathcal{C}(\mathbb{N})$, respectively. The results presented in sections 3 . and 5 ., and Theorem 5.2 , state the commutativity of the diagram in Fig. 1.

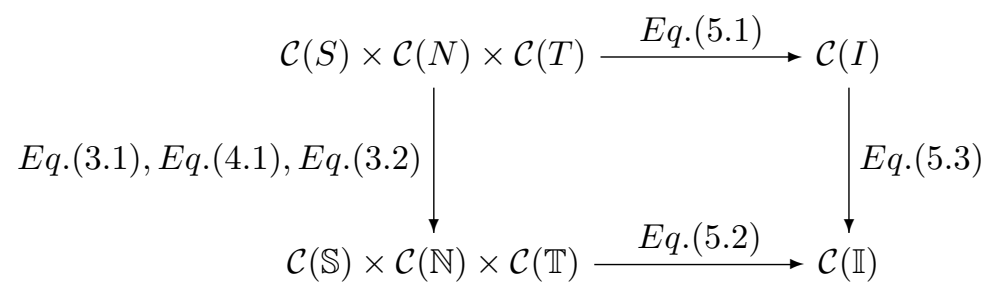

Figure 1: Classes of interval D-implications

Theorem 5.3. If $\mathbb{I}$ is an interval fuzzy $D$-implication, then the functions $\mathbb{I}, \overline{\mathbb{I}}: U^{2} \rightarrow$ $U$, defined as in Eq. (3.3) and Eq. (3.4), respectively, are D-implications.

Proof. We prove the first case. Considering $x, y \in U$ :

$$
\begin{aligned}
\mathbb{I}(x, y) & =l(\mathbb{I}([x, x],[y, y]))=l(\mathbb{S}(\mathbb{T}(\mathbb{N}([x, x]), \mathbb{N}([y, y])),[y, y])) \text { by Eq. }(3.3) \\
& =l(\mathbb{S}(\mathbb{T}([N(x), N(x)],[N(y), N(y)]),[y, y])) \text { by Eq. (4.1) and Prop. } 4.1 \\
& =l[\mathbb{S}(\mathbb{T}(N(x), N(y)), y), \overline{\mathbb{S}}(\overline{\mathbb{T}}(N(x), N(y)), y)] \text { by Eq. }(3.5), \text { Eq. }(3.6) \\
& =\mathbb{S}(\mathbb{T}(N(x), N(y)), y)=I_{\mathbb{S}, \mathbb{T}, N}(x, y) \text { by Eq. (2.1), Eq. } \quad \text { (5.1) }
\end{aligned}
$$

The second case is analogous.

The reconstruction of an interval fuzzy D-implication (i.e., the converse of Theorem 5.3) is not possible considering just the fuzzy implications $\underline{\mathbb{I}}$ and $\overline{\mathbb{I}}$. Observe Example 6. 
Example 6. Consider $\mathbb{I}_{\mathbb{S}_{L}, \mathbb{T}_{M}, \mathbb{N}_{C}}(X, Y)$ presented in Example 5(1) and the subintervals of the unit interval $[0,1], X=[0.2 ; 0.3], Y=[0.3 ; 0.5] \leq Z=[0.4 ; 0.8]$. Thus, $\mathbb{I}_{\mathbb{S}_{L}, \mathbb{T}_{M}, \mathbb{N}_{C}}(X, Y)=[0.5 ; 1.0] \geq[0.4 ; 1.0]=\mathbb{I}_{\mathbb{S}_{L}, \mathbb{T}_{M}, \mathbb{N}_{C}}(X, Z)$. That means,

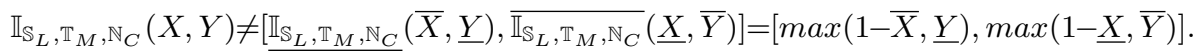

We introduce the following proposition in order to get the converse of Theorem 5.3.

Proposition 5.5. Let $\mathbb{I}$ be an interval D-implication satisfying the property $\mathbb{I} 1 b$. Then it holds that $\mathbb{I}(X, Y)=[\underline{\mathbb{I}}(\bar{X}, \underline{Y}), \overline{\mathbb{I}}(\underline{X}, \bar{Y})]$.

Proof. Since $\mathbb{S}, \mathbb{T}$ and $\mathbb{N}$ are inclusion monotonic, $\mathbb{I}_{\mathbb{S}, \mathbb{T}, \mathbb{N}}$ also is inclusion monotonic. Thus, since $\mathbb{I}$ satisfies the property $\mathbb{I} 1 b$, then from Prop. 5.4 and Theorem 5.1, it follows that $\mathbb{I}_{\mathbb{S}, \mathbb{T}, \mathbb{N}}(X, Y)=\left[\mathbb{I}_{\mathbb{S}, \mathbb{T}, \mathbb{N}}(\bar{X}, \underline{Y}), \overline{\mathbb{I}_{\mathbb{S}, \mathbb{T}, \mathbb{N}}}(\underline{X}, \bar{Y})\right]$.

\section{Interval Automorphism}

Definition 6.1. A mapping $\rho: U \longrightarrow U$ is an automorphism if it is bijective and monotonic, that is, $x \leq y \Rightarrow \rho(x) \leq \rho(y)[22,28]$.

The inverse of an automorphism is also an automorphism. Based on [11,21], the action of an automorphism $\rho$ on a function $f: U^{n} \rightarrow U$, denoted by $f^{\rho}$, is defined as

$$
f^{\rho}\left(x_{1}, \ldots, x_{n}\right)=\rho^{-1}\left(f\left(\rho\left(x_{1}\right), \ldots, \rho\left(x_{n}\right)\right)\right) .
$$

As it is well known, the action of $\rho$ preserves fuzzy connectives [30, 11], i.e., $S^{\rho}, T^{\rho}, N^{\rho}$ and $I^{\rho}$ are fuzzy t-conorm, t-norm, (strong) negation and implication, respectively.

Proposition 6.1. Let $S$ be a t-conorm, $T$ be a t-norm and $N$ be a strong negation. Then it holds that $I_{S^{\rho}, T^{\rho}, N^{\rho}}=I_{S, T, N}^{\rho}$.

Proof. Considering $x, y \in U$, one has that

$$
\begin{aligned}
I_{S^{\rho}, T^{\rho}, N^{\rho}} & =S^{\rho}\left(T^{\rho}\left(N^{\rho}(x), N^{\rho}(y)\right), y\right) \text { by Eq. }(5.1) \\
& =\rho^{-1}(S((T(N(\rho(x)), N(\rho(y)))), \rho(y)) \text { by by Eq. }(6.1) \\
& =\rho^{-1}\left(I_{S, T, N}(\rho(x), \rho(y))\right)=I_{S, T, N}^{\rho}(x, y) \text { by Eq. (5.1), Def. } 6.1
\end{aligned}
$$

Corollary 6.2. If $I$ is an D-implication then $I^{\rho}$ is also an D-implication.

Proof. It follows from the definition of D-implication and Prop. 6.1.

A mapping $\varrho: \mathbb{U} \longrightarrow \mathbb{U}$ is an interval automorphism if it is bijective and monotonic w.r.t. the product order, that is, $X \leq Y \Rightarrow \varrho(X) \leq \varrho(Y)[18,19]$.

Theorem 6.1. $\varrho: \mathbb{U} \longrightarrow \mathbb{U}$ is an interval automorphism if and only if there is an automorphism $\rho: U \rightarrow U$, such that $\varrho=\widehat{\rho}$ and

$$
\widehat{\rho}(X)=[\rho(\underline{X}), \rho(\bar{X})] .
$$


Proof. See [18, Theorem 2], [8, Theorem 5.2].

Thus, the interval automorphism $\widehat{\rho}$ is the best interval representation of the automorphism $\rho$. The action of an interval automorphism $\varrho$ on an interval function $F: \mathbb{U}^{n} \rightarrow \mathbb{U}$, defined as

$$
F^{\varrho}\left(X_{1}, X_{2}, \ldots, X_{n}\right)=\varrho^{-1}\left(F\left(\varrho\left(X_{1}\right), \varrho\left(X_{2}\right), \ldots, \varrho\left(X_{n}\right)\right)\right) .
$$

preserves interval fuzzy connectives, i.e., $\mathbb{S}^{\varrho}, \mathbb{T}^{\varrho}, \mathbb{N}^{\varrho}$ and $\mathbb{I}^{\varrho}$ are also interval fuzzy t-conorm, t-norm, (strong) negation and implication, respectively $[4,8,10]$. In the following, we apply the results concerned with the generation of new interval D-implications based on the actions of interval automorphisms.

\section{Interval Automorphisms and Interval D-impli- cations}

Firstly, the action of an interval automorphism preserving interval D-implications is shown.

Theorem 7.1. Let $\varrho: \mathbb{U} \longrightarrow \mathbb{U}$ be an interval automorphism and $\mathbb{I}: \mathbb{U}^{2} \longrightarrow \mathbb{U}$ be an interval D-implication. Then $\mathbb{I}^{\varrho}: \mathbb{U}^{2} \longrightarrow \mathbb{U}$ is also an interval D-implication.

Proof. It is sufficient to prove that the following equation holds:

$$
\mathbb{I}_{\mathbb{S}, \mathbb{T}, \mathbb{N}}^{\varrho}(X, Y)=\mathbb{S}^{\varrho}\left(\mathbb{T}^{\varrho}\left(\mathbb{N}^{\varrho}(X), \mathbb{N}^{\varrho}(Y)\right), Y\right) .
$$

Considering $X, Y \in \mathbb{U}$, we have that

$$
\begin{aligned}
\mathbb{I}_{\mathbb{S}, \mathbb{T}, \mathbb{N}}^{\varrho}(X, Y) & =\varrho^{-1}\left(\mathbb{I}_{\mathbb{S}, \mathbb{T}, \mathbb{N}}(\varrho(X), \varrho(Y))\right) \text { by Eq. }(6.3) \\
& =\varrho^{-1}(\mathbb{S}(\mathbb{T}(\mathbb{N}(\varrho(X)), \mathbb{N}(\varrho(Y))), \varrho(Y)) \text { by Eq. }(5.2) \\
& =\varrho^{-1}\left(\mathbb { S } \left(\mathbb{T}\left(\varrho\left(\varrho^{-1}(\mathbb{N}(\varrho(X)))\right), \varrho\left(\varrho^{-1}(\mathbb{N}(\varrho(Y)))\right), \varrho(Y)\right) \text { by bijectivity of } \varrho\right.\right. \\
& =\varrho^{-1}\left(\mathbb{S}\left(\varrho\left(\varrho^{-1}\left(\mathbb{T}\left(\varrho\left(\mathbb{N}^{\varrho}(X)\right), \varrho\left(\mathbb{N}^{\varrho}(Y)\right)\right)\right)\right), \varrho(Y)\right) \text { by Eq. }(6.3)\right. \\
& =\mathbb{S}^{\varrho}\left(\mathbb{T}^{\varrho}\left(\mathbb{N} \varrho(X), \mathbb{N}^{\varrho}(Y)\right), Y\right) \text { by Eq. }(6.3)
\end{aligned}
$$

According to Theorems 6.1 and 7.1, the commutative diagram pictured in Fig. 2 holds. Based on Proposition 6.1 and Theorem 7.1, (interval) D-implications and (interval) automorphisms can be seen as objects and morphisms of the category $\mathfrak{C}(\mathcal{C}(I), \operatorname{Aut}(I))(\mathfrak{C}(\mathcal{C}(\mathbb{I}), A u t(\mathbb{I})))$, respectively. In a categorical approach, the action of an interval automorphism on an interval D-implication can be conceived as a covariant functor whose application over the D-implications and automorphisms in $\mathfrak{C}(\mathcal{C}(I)$, Aut $(I))$ returns the related best interval representation in $\mathfrak{C}(\mathcal{C}(\mathbb{I})$, Aut $(\mathbb{I})$ ). Therefore, interval automorphisms can be used to deal with optimality of interval fuzzy algorithms. 


\section{Conclusion and Final Remarks}

Considering the importance of fuzzy implications studies to perform inferences in approximate reasoning and fuzzy control, the results presented in this paper contribute firstly for the general study on D-implications, whose main features have only recently appeared as subject of some important works (see, e.g., [23, 24]). However, our main interest was on the definition of interval-valued D-implications, continuing our previous works $[5,6,7,31,32]$ on the study of the the various interval-valued implication functions deriving from interval t-norms, interval t-conorms and interval negations. In this context, we proved many important properties of interval D-implications. Finally, the paper establishes the relationship between (interval) D-implications and (interval) automorphisms, showing that the action of (interval) automorphisms preserves (interval) D-implications.

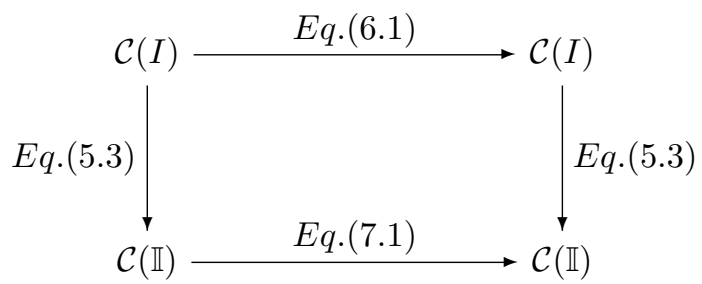

Figure 2: Commutative classes of interval D-implications and automorphisms

Acknowledgments. This work was partially supported by $\mathrm{CNPq}$ (Proc. 473201/07-0).

Resumo. Este artigo introduz os conceitos de D-implicações intervalares, analisando suas principais propriedades e correspondente relacionamento com os automorfismos intervalares.

\section{References}

[1] J. Balasubramaniam, Yager's new class of implications $J_{f}$ and some classical tautologies, Information Sciences, 177, No. 3 (2007), 930-946

[2] B.C. Bedregal, On interval fuzzy negations, Fuzzy Sets and Systems (2010) (to appear)

[3] B.C. Bedregal, G.P. Dimuro, R.H.S. Reiser, An Approach to Interval-Valued R-Implications and Automorphisms, in: "2009 IFSA World Congress/2009 EUSFLAT Conference'”, pp. 1-6, Lisboa: Instituto Superior Técnico, 2009.

[4] B.C. Bedregal, G.P. Dimuro, R.H.N. Santiago, R.H.S. Reiser, On interval fuzzy S-implications, Information Sciences (2009) (to appear).

[5] B.C. Bedregal, R.H.N. Santiago, G.P. Dimuro, R.H.S. Reiser, Interval valued R-implications and automorphisms, in "Pre-Proc. of the 2nd Workshop on Logical and Semantic Frameworks, with Applications", pp. 82-97, Ouro Preto, 2007. 
[6] B.C. Bedregal, R.H.N. Santiago, R.H.S. Reiser, G.P. Dimuro, Properties of fuzzy implications obtained via the interval constructor, TEMA Tend. Mat. Apl. Comput. 8, No. 1 (2007), 33-42.

[7] B.C. Bedregal, R.H.N. Santiago, R.H.S Reiser, G.P. Dimuro, The best interval representation of fuzzy S-implications and automorphisms, in "Proc. of the Intl. Conf. on Fuzzy Systems, 2007”, pp. 3220-3230, IEEE, Los Alamitos, 2007.

[8] B. Bedregal, A. Takahashi, The best interval representation of t-norms and automorphisms, Fuzzy Sets and Systems, 157, No. 24 (2006), 3220-3230.

[9] B. Bedregal, A. Takahashi, Interval-valued versions of t-conorms, fuzzy negations and fuzzy implications, in "Proc. of the Intl. Conf. on Fuzzy Systems, Vancouver, 2006", pp. 9553-9559, IEEE, Los Alamitos, 2006.

[10] B.C. Bedregal, A. Takahashi, Interval representations of n-dual t-conorm, in "Proc. of Logic and Applied to Tecnology" (paper n. 214), Santos, 2007.

[11] H. Bustince, P. Burillo, F. Soria, Automorphism, negations and implication operators, Fuzzy Sets and Systems, 134 (2003), 209-229.

[12] R. Callejas-Bedregal, B.C. Bedregal, Intervals as a domain constructor, TEMA Tend. Mat. Apl. Comput., 2, No. 1 (2001), 43-52.

[13] C. Carlsson, R. Fuller, "Fuzzy Reasoning in Decision Making and Optimization", Physica-Verlag Springer, Heidelberg, 2002.

[14] G. Chen, T.T. Pham, "Fuzzy Sets, Fuzzy Logic, and Fuzzy Control Systems", CRC Press, Boca Raton, 2001.

[15] D. Dubois, H. Prade, Interval-valued fuzzy sets, possibility theory and imprecise probability, in "Proc. of the Intl. Conf. on Fuzzy Logic and Technology", pp. 314-319, Barcelona, 2005.

[16] J.C. Fodor, On fuzzy implication operators, Fuzzy Sets and Systems, 42 (1991), 293-300.

[17] B.V. Gasse, C. Cornelis, G. Deschrijver, E. Kerre, On the properties of a generalized class of t-norms in interval-valued fuzzy logics, New Math. and Natural Computation, 2 (2006), 29-42.

[18] M. Gehrke, C. Walker, E. Walker, Some comments on interval valued fuzzy sets, Intl. Journal of Intelligent Systems, 11 (1996), 751-759.

[19] M. Gehrke, C. Walker, E. Walker, Algebraic aspects of fuzzy sets and fuzzy logics, in "Proc. of the Work. on Current Trends and Dev. in Fuzzy Logic", pp. 101-170, Thessaloniki, 1999.

[20] T. Hickey, Q. Ju, M. Emdem, Interval arithmetic: from principles to implementation, Journal of the ACM, 48, No. 5 (2001), 1038-1068.

[21] E. Klement, R. Mesiar, E. Pap, "Triangular Norms", Kluwer, Dordrecht, 2000. 
[22] E. Klement, M. Navara, A survey on different triangular norm-based fuzzy logics, Fuzzy Sets and Systems, 101 (1999), 241-251.

[23] M. Mas, M. Monserrat, J. Torrens, QL-implications versus D-implications, Kybernetika, 42, No. 3 (2006), 351-366.

[24] M. Mas, M. Monserrat, J. Torrens, Two types of implications derived from uninorms, Fuzzy Sets and Systems, 158, No. 3 (2007), 2612-2626.

[25] S. Mitra, S.K. Pal, Fuzzy sets in pattern recognition and machine intelligence, Fuzzy Sets and Systems, 156 (2005), 381-386.

[26] R. Moore, "Methods and Applications of Interval Analysis", SIAM, Philadelphia, 1979.

[27] R. Moore, W. Lodwick, Interval analysis and fuzzy set theory, Fuzzy Sets and Systems, 135, No. 1 (2003), 5-9.

[28] M. Navara, Characterization of measures based on strict triangular norms, Math. Analysis and Applications, 236 (1999), 370-383.

[29] H. Nguyen, V. Kreinovich, Q. Zuo, Interval-valued degrees of belief: applications of interval computations to expert systems and intelligent control, Int. J. Uncertain. Fuzziness Knowledge-Based Systems 5, No. 3 (1997), 317-358.

[30] H. Nguyen, E. Walker, "A First Course in Fuzzy Logic", Chapman \& Hall/CRC, Boca Raton, 1999.

[31] R.H.S. Reiser, G.P. Dimuro, B.C. Bedregal, R.H.N. Santiago, Interval valued QL-implications, in "Logic, Language, Information" (D. Leivant, R. Queiroz, eds.), LNCS, Vol. 4576, pp. 307-321, Springer, Berlim, 2007.

[32] R.H.S. Reiser, G.P. Dimuro, B.R.C. Bedregal, H. Santos, R.C. Bedregal, Simplications on bounded lattices and the interval constructor, TEMA Tend. Mat. Apl. Comput. 9, No. 1 (2008), 143-154.

[33] D. Ruan, E. Kerre, Fuzzy implication operators and generalized fuzzy methods of cases, Fuzzy Sets and Systems, 54 (1993), 23-37.

[34] R.H.N. Santiago, B.C. Bedregal, B. Acióly, Formal aspects of correctness and optimality in interval computations, Formal Aspects of Computing, 18, No. 2 (2006), 231-243.

[35] W. Siler, J.J. Buckley, "Fuzzy Expert Systems and Fuzzy Reasoning", John Wiley, N.Y., 2004.

[36] R. Yager, On some new classes of implication operators and their role in approximate reasoning, Information Sciences, 167 (2004), 193-216.

[37] L.A. Zadeh, Fuzzy sets, Information and Control, 8 (1965), 338-353.

[38] L.A. Zadeh, The concept of a linguistic variable and its application to approximate reasoning - I, Information Sciences, 6 (1975), 199-249. 